\title{
Latent constructs in psychosocial factors associated with cardiovascular disease: an examination by race and sex
}

\section{Cari Jo Clark ${ }^{1}$, Kimberly M. Henderson' ${ }^{1}$ Carlos F. Mendes de Leon ${ }^{2}$, Hongfei Guo ${ }^{3,4}$, Scott Lunos ${ }^{3}$, Denis A. Evans ${ }^{5,6}$ and Susan A. Everson-Rose ${ }^{1 *}$}

\author{
1 Department of Medicine, University of Minnesota Medical School, Minneapolis, MN, USA \\ ${ }^{2}$ Department of Epidemiology, University of Michigan School of Public Health, Ann Arbor, MI, USA \\ ${ }^{3}$ Biostatistical Design and Analysis Center, Clinical and Translational Science Institute, University of Minnesota, Minneapolis, MN, USA \\ ${ }^{4}$ Division of Biostatistics, University of Minnesota School of Public Health, Minneapolis, MN, USA \\ ${ }^{5}$ Department of Internal Medicine, Rush University Medical Center, Chicago, IL, USA \\ ${ }^{6}$ Rush Institute for Healthy Aging, Rush University Medical Center, Chicago, IL, USA
}

\section{Edited by:}

Katri Räikkönen, University of Helsinki, Finland

Reviewed by:

Chi-Un Pae, The Catholic University of Korea College of Medicine,

South Korea

Khemraj Hirani, University of Miami

Miller School of Medicine, USA

${ }^{*}$ Correspondence:

Susan A. Everson-Rose, Department of Medicine, University of Minnesota, 717 Delaware Street Southeast, Suite 166, Minneapolis, MN 55414, USA. e-mail:saer@umn.edu
This study examines race and sex differences in the latent structure of 10 psychosocial measures and the association of identified factors with self-reported history of coronary heart disease (CHD). Participants were 4,128 older adults from the Chicago Health and Aging Project. Exploratory factor analysis (EFA) with oblique geomin rotation was used to identify latent factors among the psychosocial measures. Multi-group comparisons of the EFA model were conducted using exploratory structural equation modeling to test for measurement invariance across race and sex subgroups. A factor-based scale score was created for invariant factor(s). Logistic regression was used to test the relationship between the factor score(s) and CHD adjusting for relevant confounders. Effect modification of the relationship by race-sex subgroup was tested. A two-factor model fit the data well (comparative fit index $=0.986$; Tucker-Lewis index $=0.969$; root mean square error of approximation $=0.039$ ). Depressive symptoms, neuroticism, perceived stress, and low life satisfaction loaded on Factor I. Social engagement, spirituality, social networks, and extraversion loaded on Factor II. Only Factor I, re-named distress, showed measurement invariance across subgroups. Distress was associated with a $37 \%$ increased odds of selfreported CHD (odds ratio: 1.37; 95\% confidence intervals: $1.25,1.50 ; p$-value $<0.0001$ ). This effect did not differ by race or sex (interaction $p$-value $=0.43$ ). This study identified two underlying latent constructs among a large range of psychosocial variables; only one, distress, was validly measured across race-sex subgroups. This construct was robustly related to prevalent $\mathrm{CHD}$, highlighting the potential importance of latent constructs as predictors of cardiovascular disease.

Keywords: psychosocial, risk factor, cardiovascular disease, factor analysis, race and sex

\section{INTRODUCTION}

Research into the influence of psychosocial risk factors on health has exploded over the past few decades. A number of psychological states and traits, acute and chronic stress, and characteristics of one's social support network have been identified as important predictors of cardiovascular disease (CVD) and disability (Rozanski et al., 1999; Everson-Rose and Lewis, 2005). However, the findings have not been entirely consistent (Everson-Rose and Lewis, 2005; Suls and Bunde, 2005). Potentially contributing to this inconsistency is the search for independent effects by modeling one psychosocial characteristic at a time or several psychosocial characteristics simultaneously in search of independent effects. This approach ignores interrelationships among psychosocial factors and potentially leads to inconsistent results (Suls and Bunde, 2005). This practice prevails despite the fact that many psychosocial factors are correlated to some degree with one another (Raynor et al., 2002; Kudielka et al., 2004; Suls and Bunde, 2005; Konttinen et al., 2008), involve considerable construct and measurement overlap (Suls and Bunde, 2005), similarly relate to poor health (Konttinen et al., 2008), and tend to cluster together in individuals (Kaplan, 1995; Williams et al., 1997b; Rozanski et al., 1999, 2005; Orth-Gomer et al., 2005). It may be a commonality among these variables rather than a distinct feature of each variable that is associated with CVD risk (Boyle et al., 2006).

Broad personality constructs such as negative affectivity may underlie a number of psychological states such as depression and anxiety (Watson and Clark, 1984; Suls and Bunde, 2005). Individual psychosocial measures may be tapping into the larger latent structure, but none measure it wholly. Hence, psychosocial risk may be modeled more accurately by composite measures that essentially pool information across variables that each tap into a facet of a potential underlying latent structure. Among the few studies that have used composite measures, most have found the presence of one or two latent constructs (Raynor et al., 2002; Harris et al., 2003; Suarez, 2003, 2006; Wilson et al., 2003; Boyle et al., 2006, 2007; Taylor et al., 2006; Lehman et al., 2009). These constructs 
have been defined in large part by a narrow range of negative psychosocial characteristics, predominately depression, anxiety, anger, and hostility. Positive psychosocial characteristics also have been modeled (Raynor et al., 2002; Harris et al., 2003; Taylor et al., 2006; Konttinen et al., 2008), but much less frequently and most commonly with only one item. Hence, the potential presence of positive latent structures has not been examined to the same extent despite research significantly relating a range of positive psychosocial characteristics to reduced risk of CVD (Kubzansky and Thurston, 2007; Steptoe et al., 2009).

In addition, the vast majority of prior studies were unable to examine potential sex and race differences in the latent structure due to the homogenous nature of their samples. This omission is significant given well-documented racial disparities in CVD. For example, the average risk of death from heart disease and stroke is 30 and $50 \%$ higher, respectively, in blacks compared to whites (Kung et al., 2008). This disparity may be attributable in part to the greater exposure of blacks to traditional CVD risk factors (e.g., hypertension; Lloyd-Jones et al., 2010; Whitfield et al., 2002) but also in part through greater exposure to psychosocial risk factors. Compared with whites, blacks have lower SES, report higher levels of hostility and depressive symptoms, and greater exposure to discrimination and negative life events (Krieger and Sidney, 1996; Williams et al., 1997a; Mustillo et al., 2004; Everson-Rose and Lewis, 2005; Krieger et al., 2005). However, a focus on racial differences alone may obscure important sex differences in psychosocial risk. Men frequently report greater levels of perceived discrimination (28-30) and hostility (Barefoot et al., 1991) and women are more likely to be depressed (Kessler et al., 1993) and distressed (33-35) but also frequently have more robust social networks than men $(36,37)$.

Therefore, further research is needed on potential latent constructs underlying psychosocial risk including potential differences by race and sex. The present study begins to fill these gaps in the literature by using factor analysis to identify the latent structure(s) of a wide range of psychosocial measures and whether these structures differ by race and sex subgroups. Differences in the level of psychosocial risk by race and sex are examined with composite measure(s) representing the identified latent factors. Finally, the ability of the composite measure(s) to predict self-reported history of coronary heart disease (CHD) is examined.

\section{MATERIALS AND METHODS \\ PARTICIPANTS}

This study utilizes data from the Chicago Health and Aging Project (CHAP), which is a longitudinal, population-based study focused on the risk of Alzheimer's disease and other chronic diseases of the elderly (Bienias et al., 2003). A door-to-door census was conducted between 1993 and 1996 in three adjacent neighborhoods in Chicago to enumerate the population $(N=64,911)$ and identify the number of residents who were 65 years or older $(n=8,509)$. Age $\geq 65$ was the only inclusion criterion for the study. Between identification during the census and the conduct of the baseline interview (1993-1997), 432 people died and 251 left the community, leaving 7,826 persons eligible for the study; a total of 6,158 $(78.7 \%)$ participated in the baseline interview. Each respondent was followed up for re-interview approximately every 3 years from his/her initial interview; presently the sixth cycle of data collection is underway. The first follow-up interview (i.e., cycle 2 data collection), was conducted between 1997 and 1999 and included the widest range of psychosocial variables; therefore, cycle 2 data were used for this analysis. The analytic sample included 4,319 adults, which represents $87.9 \%$ of the surviving participants. The sample was reduced by missing values on variables of interest leaving 4,128 for the analyses.

\section{PROCEDURE}

Data were collected through an in-home interview by trained interviewers. In addition to brief cognitive and physical function tests, participants were asked to provide demographic information and answer questions pertaining to their medical history, recent health events, health behaviors, and current psychosocial functioning. The study was approved by the Institutional Review Board of Rush University Medical Center and all participants provided written, informed consent.

\section{MEASURES}

Basic demographic information was collected including: sex (male, female), age (self-reported birth date), education (self-reported number of years of formal education completed, centered at 12), and race, which was self-reported according to the 1990 US Census categories. The vast majority of participants identified as non-Hispanic black or non-Hispanic white. Since less than $1 \%$ ( $n=56)$ of the baseline sample self-reported as Hispanic or other, these persons were included with whites in analyses. The choice of psychosocial variables to include in this study is based on (1) prior theoretical and empirical research indicating a plausible relationship between the variable and CVD $(1,39-48)$ and (2) the inclusion of the relevant measures in CHAP at cycle 2. Chosen variables include both negative and positive psychosocial characteristics.

Depressive symptoms were assessed with the Center of Epidemiologic Studies-Depression (CES-D) scale (Kohout et al., 1993). The 10-item form used in the present study was developed specifically to reduce participant burden in older adults, and its correspondence to the original version has been established (Kohout et al., 1993). The dichotomous responses to items inquiring about feelings and mental rigor during the past week were coded so that higher scores indicated greater depressive symptomatology (range $=0-10$ ). This scale demonstrated acceptable internal consistency (coefficient alpha $=0.76$ ) in this sample.

Cynicism was assessed by eight items from the Cook-Medley Hostility Scale (Cook and Medley, 1954), a commonly used measure of distrustful, suspicious attitudes. The full scale and shorter versions focused on cynicism such as in CHAP have been used widely in epidemiological studies involving middle and older aged participants (Barefoot et al., 1995; Everson et al., 1997; Everson-Rose et al., 2006). All items were true/false, with one point assigned for each "true" response; a score was created by summing across items such that higher scores indicated greater hostility (range $=0-8$ ). The eight-item scale demonstrated acceptable internal consistency in this sample (coefficient alpha $=0.73$ ).

Neuroticism was measured with four items from the NEO fivefactor inventory, a widely used measure of personality valid for 
individuals 17 years and older (Costa and McCrae, 1992). Participants reported their level of agreement on a five-point scale from strongly disagree (0) to strongly agree (4). Per previous analyses of these data, the scores were multiplied by three to correspond to the original scale (Wilson et al., 2005). Items were coded so that higher sums indicated higher levels of neuroticism (theoretical range $=0-48$, range attained in this sample was $0-45$ ). This shortened measure of neuroticism has been shown to highly correlate with the full 12-item measure in another sample of elders (Wilson et al., 2005); however, truncating the scale also results in a loss of internal consistency (coefficient alpha $=0.54$ ).

Perceived discrimination was measured by a nine-item scale that assessed subjective experiences of unfair treatment in daily life (Williams et al., 1997a). The scale was developed in a study of adults aged 18 years and older (Williams et al., 1997a) and has been used widely including in epidemiologic studies of older adults (Lewis et al., 2010). The scale does not specifically inquire whether discriminatory experiences were perceived to be in reference to any personal characteristic including race or sex. Responses were measured on a four point scale with categories ranging from never (0) to often (3). Similar to prior studies in the CHAP cohort (Lewis et al., 2009) and in other cohorts (Brown et al., 2006), responses were recoded to a binary format (often or sometimes $=1$ and rarely or never $=0$ ) and then summed across items to obtain the total score (range 0-9). The scale demonstrated good internal consistency (coefficient alpha $=0.82$ ) in this sample.

Perceived stress was measured with a six-item version of the Perceived Stress Scale (PSS), which assesses the degree to which the respondent appraises situations in the previous month to be stressful (Cohen et al., 1983). The PSS is a widely used scale to assess stress in all age groups, including older adults (Cohen and Williamson, 1988). Responses were measured on a four point scale with categories ranging from never (0) to often (3) (coefficient alpha $=0.75$ ). A score was obtained by summing across items coded so that higher scores were indicative of greater stress (range 0-18; Cohen et al., 1983).

Social network size was measured by three items derived from the Established Populations for Epidemiologic Studies of the Elderly (EPESE; Branch et al., 1986) that ask about the number of children, close relatives, and close friends seen at least once a month. Responses to the items are summed to create an overall measure of network size (range 0-98; Barnes et al., 2004a,b).

Social engagement was measured by four questions derived from the EPESE (Branch et al., 1986; Mendes De Leon et al., 2003) assessing participation in social and productive activities including: attending religious services (five-point scale from once a year or less to every day/almost every day), going to a museum (fivepoint scale from once a year or less to every day/almost every day), participation in activities or groups outside the home (five-point scale from less than once per month to five or more times per week), and employment (three-point scale including none, parttime, and full-time). As the variables were differently scaled, all were categorized into a three-level scale $(0,1$, or 2$)$ and scored similarly to previous studies using the data (Barnes et al., 2004a,b) by summing across the items with higher total scores indicating higher social engagement (range 0-7, maximum of 8 was not obtained in this sample). As the measure was not intended to represent a latent construct (Barnes et al., 2004a) no coefficient alpha was computed.

Spirituality was assessed with items derived primarily from the EPESE (Idler, 1987; Koenig and Bussing, 2010) measuring salience of faith, strength, and comfort from religion, and frequency of prayer. Both salience of faith and strength/comfort were rated on a four point scale, from not at all (0) to very (3). Frequency of prayer was rated on a five-point scale, from once a year or less (0) to every day or almost every day (4). Items were coded so that higher summed scores were indicative of greater spirituality (range $=0$ 10). This measure demonstrated good internal reliability in this sample (coefficient alpha $=0.89$ ).

Life satisfaction was adapted from a measure used in the Enhancing Recovery in Coronary Heart Disease study (Mendes De Leon et al., 2006). Items were selected for inclusion in the CHAP study based on their relevance to older adults. Participants reported their level of agreement on a four point scale from strongly disagree (0) to strongly agree (3) with higher total scores representing higher satisfaction (range $=0-15$ ). The scale demonstrated acceptable internal consistency (coefficient alpha $=0.73$ ) in this sample.

Extraversion was measured with four items from the Neo fivefactor inventory, a widely used measure of personality valid for individuals 17 years of age or older (Costa and McCrae, 1992). Participants reported their level of agreement on a five-point scale from strongly disagree ( 0 ) to strongly agree (4). Per previous analyses of these data, the scores were multiplied by three to correspond to the original scale (Wilson et al., 2005). Items were coded so that higher sums indicated higher levels of extraversion (theoretical range $=0-48$, range attained in this sample 3-48). Similar to neuroticism, this shortened measure of extraversion has been shown to highly correlate with the full 12-item measure in another sample of elders (Wilson et al., 2005); however, truncating the items also results in a loss of internal consistency (coefficient alpha $=0.30$ ).

Coronary heart disease was measured using a standard question derived from the EPESE asking participants to report whether they had been diagnosed by a health care provider as having CHD (heart attack or coronary, coronary thrombosis, coronary occlusion, or myocardial infarction; Farmer et al., 1986) since the last visit. For analysis, a participant was considered to have a history of $\mathrm{CHD}$ if s/he answered affirmatively to this question at either the first or second cycle of data collection in CHAP.

\section{STATISTICAL ANALYSIS}

Descriptive statistics and correlation matrices were calculated for the psychosocial measures. Exploratory factor analysis (EFA) with oblique geomin rotation was used to identify latent factors. Because several of the variables were not normally distributed (discrimination, social network, spirituality, and depressive symptoms), all models were tested using a robust maximum likelihood estimator (MLR), which provides SEs and tests of fit that are robust against non-normality (Yuan and Bentler, 2000). Criteria for determining the number of factors retained included an Eigenvalue $>1.0$, a distinct break in slope of the Scree Plot, and at least three items loading on a factor (Costello and Osborne, 2005). Meaningful loadings were determined by a value of $\geq 0.30$ with the highest meaningful loading determining the variable's 
placement on a factor. All models were tested using Mplus 5.21 software (Muthén and Muthén, 1998-2009).

As recommended (Kline, 2005; Raykov and Marcoulides, 2006), various fit indices were used to determine the fit of the model. In this study, we use the comparative fit index (CFI), Tucker-Lewis index (TLI), and the root mean square error of approximation (RMSEA), all of which are sample size independent. Values for CFI and TLI that are $>0.90$ have been deemed a good fit (McDonald and Ho, 2002; Kline, 2005); although stricter cutoffs (0.95) have also been proposed (Hu and Bentler, 1999). Therefore, we consider 0.90 as the cutoff for acceptable fit and 0.95 as the cutoff for good fit. RMSEA values less than or equal to 0.06 are considered good fit, and less than or equal to 0.08 are acceptable (Hair et al., 1998). The chi-square test statistic is also reported, but it was not used to predict model fit since it is sensitive to large sample sizes and model complexity.

Multi-group comparisons of the EFA model were conducted using exploratory structural equation modeling (ESEM) to determine whether the model was similar across race-sex subgroups. ESEM is a recent tool available in Mplus (Asparouhov and Muthén, 2009) which merges the statistical capabilities of EFA, confirmatory factor analysis, and structural equation modeling, and therefore provides the most appropriate conditions for testing measurement invariance in an exploratory framework (Marsh et al., 2010). Measurement invariance was evaluated using the CFI difference test ( $\Delta$ CFI; Cheung and Rensvold, 2002), which indicates measurement invariance across groups if the difference between the CFI of the constrained and the unconstrained model is less than 0.01. Comparisons of each race-sex subgroup combination were made for both configural invariance, which imposes no invariance constraints, and metric invariance, which constrains factor loadings to be equal across groups. Metric invariance ensures that the latent factors are measured the same in each subgroup, which is necessary to create factor-based scale scores (Wu et al., 2007).

After measurement invariance was established, a factor-based scale score (Pett et al., 2003) was created by taking the average of the psychosocial scales ( $z$-scored) that loaded on that factor. The score was scaled by its SD. When necessary, scale scores were reversed before averaging. Differences in mean scale scores across race-sex subgroups were tested using an analysis of variance test. Scheffe's test was used to examine statistical differences between the various pairs of race-sex subgroups. The association between the factor score and CHD was examined with logistic regression. Both bivariate and multivariable models were constructed. The multivariable model adjusted for age, race-sex categories (white men as the reference), education, systolic blood pressure, physical activity, smoking status, and body mass index. An interaction was tested between the factor score and the race-sex subgroup variable to ascertain whether the relationship between the factor score and CHD was similar across race-sex subgroups. SAS version 9.2 was used for these analyses.

\section{RESULTS}

The sample was comprised of $38 \%$ black females $(n=1555)$, $24 \%$ white females $(n=995), 24 \%$ black males $(n=908)$, and $14 \%$ white males $(n=598)$. Participants were aged $68-105$ years ( mean $=77, \mathrm{SD}=6$ ). On average, respondents reported 12 years of formal education $(\mathrm{SD}=4)$. Descriptive statistics and a correlation matrix of the psychosocial variables are presented in Table 1.

In the EFA, a three factor solution was a better fit than a twoor one-factor model based on having the highest CFI and TLI and the lowest RMSEA (Table 2).

The factor loadings are presented in Table 3. Factor I was comprised of depression, neuroticism, perceived stress, and low life

Table 1 | Correlation matrix, means, and SD of the psychosocial variables $(N=4128)$.

\begin{tabular}{|c|c|c|c|c|c|c|c|c|c|c|c|c|}
\hline & & 1 & 2 & 3 & 4 & 5 & 6 & 7 & 8 & 9 & 10 & Mean (SD)* \\
\hline 1 & Perceived stress & 1.00 & & & & & & & & & & $5.44(3.66)$ \\
\hline 2 & Perceived discrimination & 0.32 & 1.00 & & & & & & & & & $1.02(1.60)$ \\
\hline 3 & Social network & -0.12 & -0.05 & 1.00 & & & & & & & & $6.93(5.95)$ \\
\hline 4 & Social engagement & -0.23 & -0.08 & 0.26 & 1.00 & & & & & & & $2.02(1.61)$ \\
\hline 5 & Spirituality & -0.07 & 0.01 & 0.15 & 0.26 & 1.00 & & & & & & $9.01(2.05)$ \\
\hline 6 & Life satisfaction & -0.41 & -0.12 & 0.20 & 0.36 & 0.12 & 1.00 & & & & & $8.89(2.33)$ \\
\hline 7 & Depressive symptoms & 0.52 & 0.24 & -0.15 & -0.29 & -0.08 & -0.48 & 1.00 & & & & $1.77(2.13)$ \\
\hline 8 & Cynicism & 0.20 & 0.37 & -0.07 & -0.14 & -0.01 & -0.09 & 0.11 & 1.00 & & & $2.91(2.14)$ \\
\hline 9 & Neuroticism & 0.46 & 0.25 & -0.14 & -0.22 & -0.02 & -0.42 & 0.49 & 0.22 & 1.00 & & $16.76(7.29)$ \\
\hline 10 & Extraversion & -0.14 & -0.08 & 0.18 & 0.20 & 0.13 & 0.25 & -0.12 & -0.15 & -0.12 & 1.00 & $25.67(6.53)$ \\
\hline
\end{tabular}

*Variables were z-scored for analysis.

Table 2 | Exploratory factor analysis model fit statistics $(N=4128)$.

\begin{tabular}{|c|c|c|c|c|c|c|}
\hline Model & $\chi^{2}$ & df & $p$ & CFI & TLI & RMSEA (90\% Cls) \\
\hline 1 Factor & 1559.732 & 35 & $<0.001$ & 0.776 & 0.713 & $0.103(0.098,0.107)$ \\
\hline 2 Factor & 950.589 & 26 & $<0.001$ & 0.864 & 0.765 & $0.093(0.088,0.098)$ \\
\hline 3 Factor & 215.988 & 18 & $<0.001$ & 0.971 & 0.927 & $0.052(0.046,0.058)$ \\
\hline
\end{tabular}


Table 3 | Factor loadings for the three factor model $(N=4128)$.

\begin{tabular}{|c|c|c|c|c|c|c|}
\hline \multirow[t]{2}{*}{ Psychosocial variables } & \multicolumn{2}{|c|}{$\mathbf{I}$} & \multicolumn{2}{|c|}{ II } & \multicolumn{2}{|c|}{ III } \\
\hline & Loading & SE & Loading & SE & Loading & SE \\
\hline Depressive symptoms & 0.76 & 0.02 & 0.00 & 0.02 & 0.04 & 0.04 \\
\hline Neuroticism & 0.63 & 0.02 & 0.00 & 0.02 & 0.15 & 0.03 \\
\hline Perceived stress & 0.65 & 0.04 & 0.00 & 0.01 & 0.26 & 0.04 \\
\hline Life satisfaction & -0.54 & 0.02 & 0.26 & 0.02 & 0.05 & 0.03 \\
\hline Spirituality & 0.09 & 0.04 & 0.41 & 0.03 & 0.01 & 0.02 \\
\hline Social engagement & -0.12 & 0.05 & 0.58 & 0.04 & -0.01 & 0.01 \\
\hline Social network & 0.02 & 0.03 & 0.40 & 0.03 & -0.03 & 0.02 \\
\hline Extraversion & 0.00 & 0.01 & 0.39 & 0.03 & -0.11 & 0.03 \\
\hline Cynicism & -0.01 & 0.01 & -0.22 & 0.05 & 0.53 & 0.06 \\
\hline Perceived discrimination & 0.14 & 0.07 & 0.01 & 0.01 & 0.66 & 0.07 \\
\hline
\end{tabular}

Item placement indicated by bold font.

satisfaction. Factor II was comprised of social engagement, spirituality, social networks, and extraversion. Factor III was comprised of hostility and perceived discrimination. Factor I and II were moderately correlated $(-0.44)$. Factor III was poorly correlated with Factor I (0.10) and II (0.09). Since Factor III was comprised of only two measures (perceived discrimination and hostility), and thus did not meet our criteria for determining factors, its measures were removed from the analysis.

The EFA was re-run on the remaining eight variables. A two-factor model fit the data well $(\mathrm{CFI}=0.986$; $\mathrm{TLI}=0.969$; RMSEA $=0.039)$. Its structure re-created two of the factors from the initial three factor model. The factors were moderately correlated with one another $(-0.50)$. The two-factor model, however, did not fit all race-sex subgroups equally well (Table 4).

A formal test of measurement invariance confirmed this finding (Table 5). The configural and metric models for all subgroup comparisons fit the data well. The CFI difference test showed factorial invariance only between black and white men, and white men and women. From a visual inspection of the models, most of the differences in factor loadings were among items loading on Factor II. Therefore, further analysis to examine the suitability of creating factor-based scale scores was restricted to Factor I. An ESEM of this factor (i.e., a one-factor model) showed measurement invariance across all subgroups, except for a borderline CFI difference between black and white women $(-0.012)$. Therefore a factor-based scale score was created for Factor I, which, due to its constituent items was re-named distress.

Table 6 summarizes the race-sex subgroup differences in distress scores. Blacks had higher levels of distress, with black women having significantly higher levels of distress than all other racesex subgroups. Black men had higher levels of distress than white women, but these differences were not statistically different. White men had significantly lower levels of distress than any other race-sex subgroup.

In the sample, $16.38 \%(n=676)$ reported a history of CHD. In a model controlling for age, race-sex subgroups, education, systolic blood pressure, physical activity, smoking status, and body mass index, distress was associated with a $37 \%$ increased odds of self-reported CHD (odds ratio: 1.37; 95\% confidence intervals:
$1.25,1.50 ; p$-value $<0.0001)$. The test for an interaction between distress and the race-sex subgroup variable was not significant ( $p$ value $=0.43$ ) indicating that the relationship between distress and CHD was not different by race or sex.

\section{DISCUSSION}

This study examined race and sex differences in the latent structure of a wide range of psychosocial measures. We found that while the same factor structure was present among all race-sex subgroups, the factors were not measured equally well by the items modeled. Among the factors identified, only one, distress, was similarly measured across race-sex subgroups. Race and sex differences in the levels of distress were observed, but these differences did not translate into significantly different relationships between distress and self-reported CHD.

While three factors were originally identified, only two were comprised of enough individual measures to constitute a factor. These two factors appeared to represent negative and positive psychosocial characteristics. The distress factor may represent a larger construct of negative affectivity. Individuals high in negative affectivity are more likely to report distress, discomfort, and dissatisfaction (Watson and Clark, 1984). This was borne out in this study in that neuroticism, perceived stress, depressive symptoms, and low life satisfaction loaded together on the distress factor. The distinction between the distress factor and the majority of the positive psychosocial measures is supported by research that has shown that positive psychosocial characteristics are related to one another independently of negative affect and negative psychosocial characteristics are related to one another independently of positive affect (Steptoe et al., 2008). A prior factor analysis of psychosocial predictors of cardiac mortality found that the positive characteristics loaded on a factor that was distinct from the negative psychosocial characteristics (Frasure-Smith and Lesperance, 2003). The distinction is also supported by theoretical and empirical research demonstrating that positive personality traits, under the broad umbrella of extraversion, lead to positive affect whereas negative personality traits, under the broad umbrella of neuroticism, lead to negative affect (Costa and McCrae, 1980). While this study is cross-sectional and thus cannot address temporal differences, the 
Table 4 | Factor loadings and model fit by race-sex subgroups.

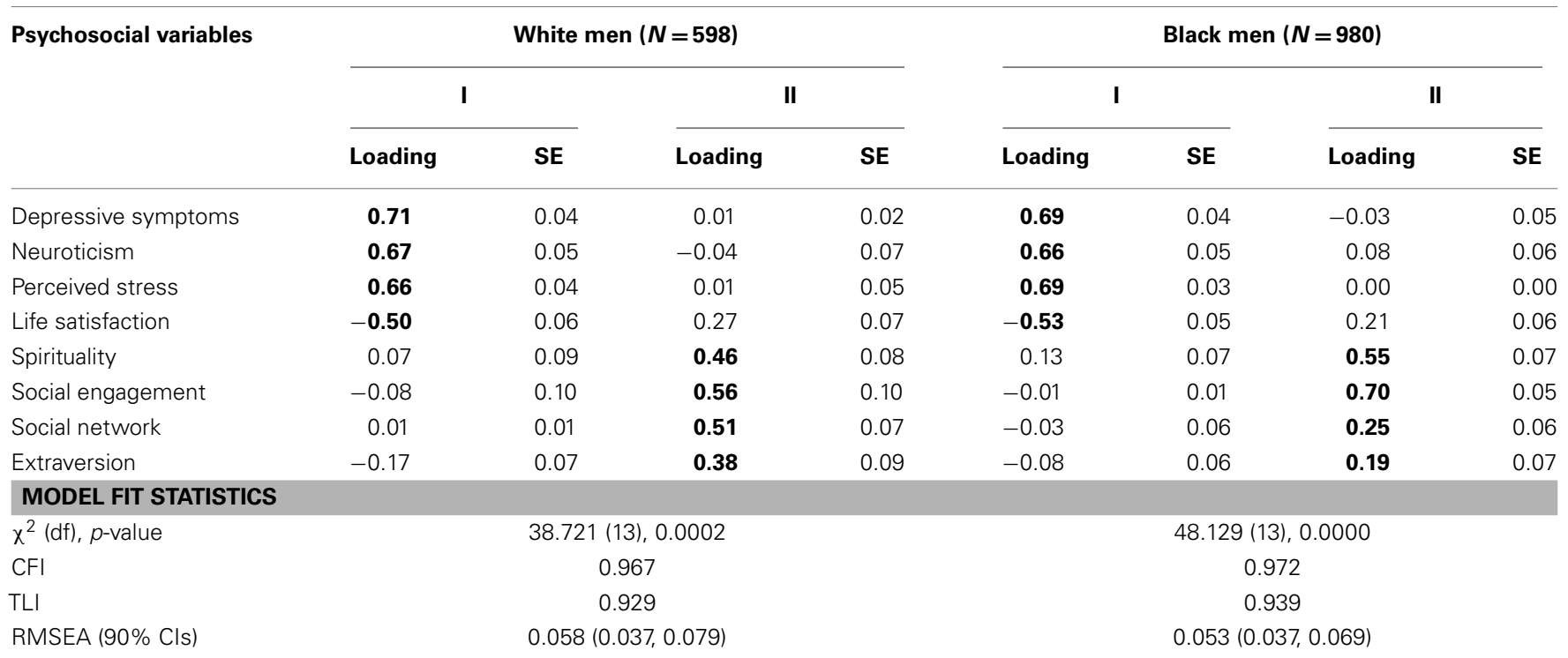

\begin{tabular}{|c|c|c|c|c|c|c|c|c|}
\hline \multirow[b]{2}{*}{ Depressive symptoms } & \multicolumn{4}{|c|}{ White women $(N=995)$} & \multicolumn{4}{|c|}{ Black women $(N=1,555)$} \\
\hline & 0.68 & 0.05 & -0.07 & 0.06 & 0.76 & 0.04 & -0.05 & 0.05 \\
\hline Neuroticism & 0.64 & 0.03 & -0.01 & 0.00 & 0.71 & 0.02 & 0.00 & 0.00 \\
\hline Life satisfaction & -0.41 & 0.05 & 0.37 & 0.06 & -0.43 & 0.05 & 0.35 & 0.05 \\
\hline Spirituality & 0.11 & 0.06 & 0.44 & 0.05 & 0.03 & 0.04 & 0.33 & 0.04 \\
\hline Extraversion & -0.05 & 0.06 & 0.44 & 0.06 & 0.06 & 0.05 & 0.34 & 0.06 \\
\hline \multicolumn{9}{|c|}{ MODEL FIT STATISTICS } \\
\hline$\chi^{2}(\mathrm{df}), p$-value & \multicolumn{4}{|c|}{$31.342(13), 0.0030$} & \multicolumn{4}{|c|}{20.370 (13), 0.0864} \\
\hline $\mathrm{CFI}$ & \multicolumn{4}{|c|}{0.987} & \multicolumn{4}{|c|}{0.997} \\
\hline TLI & \multicolumn{4}{|c|}{0.972} & \multicolumn{4}{|c|}{0.993} \\
\hline
\end{tabular}

clustering of negative psychosocial constructs with neuroticism and positive psychosocial constructs with extraversion is theoretically and empirically defensible. However, factor-based scale scores were only calculated for the measures loading on the distress factor. It was the only latent factor similarly measured across racesex subgroups suggesting that it is a robust measure of negative psychosocial risk.

\section{STRENGTHS AND LIMITATIONS}

These findings must be considered in light of the study's limitations. This study was unable to include some psychosocial risk factors for CVD (e.g., anxiety and anger) that have been examined in previous factor analyses. Some potentially relevant personality constructs such as openness, and stressors of particular relevance to older adults, such as caregiving, could not be examined. Caregiving in particular was not included in the present study since only $8 \%$ of the analytic sample was providing unpaid care at the time of data collection for cycle 2 , rendering the variable of limited utility to the analysis. Given the unique range of variables included in this study, it is difficult to know how stable the underlying constructs are to the inclusion of other relevant but unexamined psychosocial variables. Additional research incorporating these variables is needed to clarify this. Further, the analyses were conducted on a cohort of elderly black and white participants in select neighborhoods in Chicago which might impact the generalizability of the findings. Finally, additional research is needed to test the predictive power of the distress construct in a longitudinal framework with objectively defined cardiovascular endpoints.

This study also has notable strengths. The study utilized a population-based design and included sufficient numbers of men, women and white and black participants to examine possible differences in factor structure and factor scale score by race and sex. We used robust, state-of-the-art statistical tools and included a wider array of psychosocial measures than in prior research.

\section{CONCLUSION}

This study found that two latent constructs were identifiable in an exploratory investigation of a large range of psychosocial variables but that only one of these constructs, distress, was validly measured across race-sex subgroups. Therefore, caution is warranted 
Table 5 | Measurement invariance tests between race-sex subgroup pairs, two-factor model.

\begin{tabular}{|c|c|c|c|c|c|}
\hline Model & $\chi^{2}$ (df) & CFI & $\Delta \mathrm{CFI}$ & TLI & RMSEA $(90 \% \mathrm{Cl})$ \\
\hline \multicolumn{6}{|c|}{ BLACK MEN AND BLACK WOMEN } \\
\hline Configural invariance & $68.115(26)^{*}$ & 0.988 & - & 0.973 & $0.036(0.026-0.046)$ \\
\hline \multicolumn{6}{|c|}{ BLACK MEN AND WHITE MEN } \\
\hline Configural invariance & $86.846(26)^{*}$ & 0.970 & - & 0.935 & $0.054(0.042-0.067)$ \\
\hline Configural invariance & $80.185(26)^{*}$ & 0.980 & - & 0.956 & $0.046(0.035-0.058)$ \\
\hline Metric invariance & $148.813(38)^{*}$ & 0.958 & -0.022 & 0.938 & $0.054(0.045-0.064)$ \\
\hline \multicolumn{6}{|c|}{ BLACK WOMEN AND WHITE WOMEN } \\
\hline Configural invariance & $51.093(26)^{*}$ & 0.933 & - & 0.985 & $0.028(0.016-0.039)$ \\
\hline Metric invariance & $125.351(38)^{*}$ & 0.975 & -0.018 & 0.963 & $0.042(0.034-0.051)$ \\
\hline Configural invariance & $70.373(26)^{*}$ & 0.980 & - & 0.956 & $0.046(0.033-0.059)$ \\
\hline Metric invariance & $94.290(38)^{*}$ & 0.974 & -0.006 & 0.962 & $0.043(0.032-0.054)$ \\
\hline
\end{tabular}

${ }^{*} p$-value $<0.05$.

Table 6 | Mean distress by race-sex subgroup $(N=4128)$.

\begin{tabular}{|c|c|c|c|c|}
\hline & & Mean & SD & Different from* \\
\hline a. & Black women & 0.18 & 1.05 & $b, c, d$ \\
\hline b. & Black men & 0.01 & 1.02 & $a, d$ \\
\hline c. & White women & -0.08 & 0.94 & $a, d$ \\
\hline d. & White men & -0.35 & 0.84 & $a, b, c$ \\
\hline
\end{tabular}

${ }^{*}$ Denotes statistically significant differences at the $p$-value $<0.05$.

when creating composite measures since it can not be assumed that the constructs are similarly measured in all participants. However, preliminary evidence from this study suggests that distress may be robustly related to prevalent $\mathrm{CHD}$, highlighting the potential importance of latent constructs as predictors of CVD. As a composite measure, it more closely represents the way that

\section{REFERENCES}

Asparouhov, T., and Muthén, B. (2009). Exploratory structural equation modeling. Struct. Equ. Modeling $16,397-438$.

Barefoot, J. C., Larsen, S., Von Der Lieth, L., and Schroll, M. (1995). Hostility, incidence of acute myocardial infarction, and mortality in a sample of older Danish men and women. Am. J. Epidemiol. 142, 477-484.

Barefoot, J. C., Peterson, B. L., Dahlstrom, W. G., Siegler, I. C., Anderson, N. B., and Williams, R. B. Jr. (1991). Hostility patterns and health implications: correlates of Cook-Medley hostility scale scores in a national survey. Health Psychol. $10,18-24$

Barnes, L. L., Mendes De Leon, C. F., Bienias, J. L., and Evans, D. A. (2004a). A longitudinal study of black-white differences in social resources. J. Gerontol. B. Psychol. Sci. Soc. Sci. 59, S146-153.

Barnes, L. L., Mendes De Leon, C. F., Wilson, R. S., Bienias, J. L., and Evans, D. A. (2004b). Social resources and cognitive decline in a population of older African Americans and whites. Neurology 63, 2322-2326.

Bienias, J. L., Beckett, L. A., Bennett, D. A., Wilson, R. S., and Evans, D. A. (2003). Design of

many negative psychosocial characteristics occur in nature. Further research is needed to ascertain whether composite indicators of psychosocial distress consistently and accurately predict a range of cardiovascular disease endpoints.

\section{ACKNOWLEDGMENTS}

This work was supported by grants HL084209, AG11101, and ES10902 from the National Institutes of Health. Additional support was provided by the Program in Health Disparities Research and the Applied Clinical Research Program (Drs. Clark and Everson-Rose) at the University of Minnesota. The authors thank all participants in the Chicago Health and Aging Project, Ann Marie Ryan Stewart for community development and oversight of project coordination, Michelle Bos, Flavio Lamorticella, Jennifer Tarpey, and their staff for study coordination and data collection.

the Chicago Health and Aging Project (CHAP). J. Alzheimers Dis. 5, 349-355.

Boyle, S. H., Jackson, W. G., and Suarez, E. C. (2007). Hostility, anger, and depression predict increases in $\mathrm{C} 3$ over a 10-year period. Brain Behav. Immun. 21, 816-823.

Boyle, S. H., Michalek, J. E., and Suarez, E. C. (2006). Covariation of psychological attributes and incident coronary heart disease in U.S. Air Force veterans of the Vietnam war. Psychosom. Med. 68, 844-850.

Branch, L. G., Berkman, L. F., Brock, D. B., Cosmatos, D., Keough, M. E. Lemke, J. H., Mcgloin, J., and Morris, M. C. (1986). "Social functioning," in Established Populations for Epidemiologic Studies of the Elderly Resource Data Book, eds J. CornoniHuntley, D. B. Brock, A. M. Ostfeld, J. O. Taylor, and R. B. Wallace (Washington, DC: US Department of Health and Human Services), 33-55.

Brown, C., Matthews, K. A., Bromberger, J. T., and Chang, Y. (2006). The relation between perceived unfair treatment and blood pressure in a racially/ethnically diverse sample of women. Am. J. Epidemiol. 164, 257-262.

Cheung, G. W., and Rensvold, R. B. (2002). Evaluating goodness-of-fit indexes for testing measurement 
invariance. Struct. Equ. Modeling 9, 233-255.

Cohen, S., Kamarck, T., and Mermelstein, R. (1983). A global measure of perceived stress. J. Health Soc. Behav. 24, 385-396.

Cohen, S., and Williamson, G. (1988). "Perceived stress in a probability sample of the U.S.," in The Social Psychology of Health: Claremont Symposium on Applied Social Psychology, eds S. Spacapam and S. Oskamp (Newbury Park, CA: Sage), 31-67.

Cook, W., and Medley, D. (1954). Proposed hostility and pharisaic virtue scales for the MMPI. J. Appl. Psychol. 38, 414-418.

Costa, P. T. Jr., and McCrae, R. R. (1980). Influence of extraversion and neuroticism on subjective well-being: happy and unhappy people. J. Pers. Soc. Psychol. 38, 668-678.

Costa, P. T., and McCrae, R. R. (1992). Revised NEO Personality Inventory (NEO-PI-R) and NEO Five-Factor Inventory (NEO-FFI) Professional Manual. Odessa, FL: Psychological Assessment Resources.

Costello, A. B., and Osborne, J. (2005). Best practices in exploratory factor analysis: four recommendations for getting the most from your analysis. Pract. Assess. Res. Eval. 10, 1-9.

Everson, S. A., Kauhanen, J., Kaplan, G. A., Goldberg, D. E., Julkunen, J., Tuomilehto, J., and Salonen, J. T. (1997). Hostility and increased risk of mortality and acute myocardial infarction: the mediating role of behavioral risk factors. Am. J. Epidemiol. 146, 142-152.

Everson-Rose, S. A., and Lewis, T. T. (2005). Psychosocial factors and cardiovascular diseases. Annu. Rev. Public Health 26, 469-500.

Everson-Rose, S. A., Lewis, T. T., Karavolos, K., Matthews, K. A., SuttonTyrrell, K., and Powell, L. H. (2006). Cynical hostility and carotid atherosclerosis in African American and white women: the Study of Women's Health Across the Nation (SWAN) Heart Study. Am. Heart J. 152, 982.e7-982.e13.

Farmer, M. E., Ostfeld, A. M., CornoniHuntley, J., Taylor, J. O., Wallace, R. B., and White, L. R. (1986). "Chronic conditions," in Established Populations for Epidemiologic Studies of the Elderly Resource Data Book, eds J. Cornoni-Huntley, D. B. Brock, A. M. Ostfeld, J. O. Taylor, and R. B. Wallace (Washington, DC: US Department of Health and Human Services), 95-128.

Frasure-Smith, N., and Lesperance, F. (2003). Depression and other psychological risks following myocardial infarction. Arch. Gen. Psychiatry 60, 627-636.

Hair, J. F., Anderson, R. E., Tatham, R. L., and Black, W. G. (1998). Multivariate Data Analysis. Upper Saddle River, NJ: Prentice Hall.

Harris, K. F., Matthews, K. A., SuttonTyrrell, K., and Kuller, L. H. (2003). Associations between psychological traits and endothelial function in postmenopausal women. Psychosom. Med. 65, 402-409.

Hu, L., and Bentler, P. M. (1999). Cutoff criteria for fit indexes in covariance structure analysis: conventional criteria verses new alternatives. Struct. Equ. Modeling 6, 1-55.

Idler, E. L. (1987). Religious involvement and the health of the elderly: some hypotheses and an initial test. Social Forces 66, 226-238.

Kaplan, G. A. (1995). Where do shared pathways lead? Some reflections on a research agenda. Psychosom. Med. 57, 208-212.

Kessler, R. C., Mcgonagle, K. A., Swartz, M., Blazer, D. G., and Nelson, C. B. (1993). Sex and depression in the National Comorbidity Survey. I: lifetime prevalence, chronicity and recurrence. J. Affect. Disord. 29, 85-96.

Kline, R. B. (2005). Principles and Practice of Structural Equation Modeling. New York, NY: The Guilford Press.

Koenig, H. G., and Bussing, A. (2010). The Duke University Religion Index (DUREL): a five-item measure for use in epidemiological studies. Religions 1, 78-85.

Kohout, F. J., Berkman, L. F., Evans, D. A., and Cornoni-Huntley, J. (1993). Two shorter forms of the CES-D (Center for Epidemiological Studies Depression) depression symptoms index. J. Aging Health 5, 179-193.

Konttinen, H., Haukkala, A., and Uutela, A. (2008). Comparing sense of coherence, depressive symptoms and anxiety, and their relationships with health in a population-based study. Soc. Sci. Med. 66, 2401-2412.

Krieger, N., and Sidney, S. (1996). Racial discrimination and blood pressure: the CARDIA study of young black and white adults. Am. J. Public Health 86, 1370-1378.

Krieger, N., Smith, K., Naishadham, D., Hartman, C., and Barbeau, E. M. (2005). Experiences of discrimination: validity and reliability of a self-report measure for population health research on racism and health. Soc. Sci. Med. 61, 1576-1596.

Kubzansky, L. D., and Thurston, R. C. (2007). Emotional vitality and incident coronary heart disease: benefits of healthy psychological functioning. Arch. Gen. Psychiatry 64, 1393-1401.

Kudielka, B. M., Von Kanel, R., Gander, M. L., and Fischer, J. E. (2004). The interrelationship of psychosocial risk factors for coronary artery disease in a working population: do we measure distinct or overlapping psychological concepts? Behav. Med. 30, 35-43.

Kung, H. C., Hoyert, D. L., Xu, J., and Murphy, S. L. (2008). Deaths: final data for 2005. Natl. Vital Stat. Rep. $56,1-120$.

Lehman, B. J., Taylor, S. E., Kiefe, C. I., and Seeman, T. E. (2009). Relationship of early life stress and psychological functioning to blood pressure in the CARDIA study. Health Psychol. 28, 338-346.

Lewis, T. T., Aiello, A. E., Leurgans, S., Kelly, J., and Barnes, L. L. (2010). Self-reported experiences of everyday discrimination are associated with elevated C-reactive protein levels in older African-American adults. Brain Behav. Immun. 24, 438-443.

Lewis, T. T., Barnes, L. L., Bienias, J. L., Lackland, D. T., Evans, D. A., and Mendes De Leon, C. F. (2009). Perceived discrimination and blood pressure in older African American and white adults. J. Gerontol. A Biol. Sci. Med. Sci. 64, 1002-1008.

Lloyd-Jones, D., Adams, R. J., Brown, T. M., Carnethon, M., Dai, S., De Simone, G., Ferguson, T. B., Ford, E., Furie, K., Gillespie, C., Go, A., Greenlund, K., Haase, N., Hailpern, S., Ho, P. M., Howard, V., Kissela, B., Kittner, S., Lackland, D., Lisabeth, L., Marelli, A., Mcdermott, M. M., Meigs, J., Mozaffarian, D., Mussolino, M., Nichol, G., Roger, V. L., Rosamond, W., Sacco, R., Sorlie, P., Thom, T. Wasserthiel-Smoller, S., Wong, N. D., and Wylie-Rosett, J. (2010). Heart disease and stroke statistics - 2010 update: a report from the American Heart Association. Circulation 121, e46-e215.

Marsh, H. W., Ludtke, O., Muthen, B., Asparouhov, T., Morin, A. J., Trautwein, U., and Nagengast, B. (2010). A new look at the big five factor structure through exploratory structural equation modeling. Psychol. Assess. 22, 471-491.

McDonald, R. P., and Ho, M. H. (2002). Principles and practice in reporting structural equation analyses. Psychol. Methods 7, 64-82.

Mendes De Leon, C. F., Czajkowski, S. M., Freedland, K. E., Bang, H., Powell, L. H., Wu, C., Burg, M. M., Dilillo, V., Ironson, G., Krumholz, H. M., Mitchell, P., and Blumenthal, J. A.
(2006). The effect of a psychosocial intervention and quality of life after acute myocardial infarction: the Enhancing Recovery in Coronary Heart Disease (ENRICHD) clinical trial. J. Cardiopulm. Rehabil. 26, 9-13; quiz 14-15.

Mendes De Leon, C. F., Glass, T. A., and Berkman, L. F. (2003). Social engagement and disability in a community population of older adults: the New Haven EPESE. Am. J. Epidemiol. 157, 633-642.

Mustillo, S., Krieger, N., Gunderson, E. P., Sidney, S., Mccreath, H., and Kiefe, C. I. (2004). Self-reported experiences of racial discrimination and black-white differences in preterm and low-birthweight deliveries: the CARDIA study. Am. J. Public Health 94, 2125-2131.

Muthén, L. K., and Muthén, B. O. (19982009). Mplus User's Guide. Los Angeles, CA: Muthén and Muthén.

Orth-Gomer, K., Albus, C., Bages, N., Debacker, G., Deter, H. C., Herrmann-Lingen, C., Oldenburg, B., Sans, S., Williams, R. B., and Schneiderman, N. (2005). Psychosocial considerations in the European guidelines for prevention of cardiovascular diseases in clinical practice: third joint task force. Int. J. Behav. Med. 12, 132-141.

Pett, M. A., Lackey, N. R., and Sullivan, J. J. (2003). Making Sense of Factor Analysis. Thousand Oaks, CA: Sage Publications.

Raykov, T., and Marcoulides, G. A. (2006). A First Course in Structural Equation Modeling. Mahwah, NJ: Lawrence Erlbaum Associates, Inc.

Raynor, D. A., Pogue-Geile, M. F., Kamarck, T. W., Mccaffery, J. M., and Manuck, S. B. (2002). Covariation of psychosocial characteristics associated with cardiovascular disease: genetic and environmental influences. Psychosom. Med. 64, 191-203; discussion 204-205.

Rozanski, A., Blumenthal, J. A., Davidson, K. W., Saab, P. G., and Kubzansky, L. (2005). The epidemiology, pathophysiology, and management of psychosocial risk factors in cardiac practice: the emerging field of behavioral cardiology. J. Am. Coll. Cardiol. 45, 637-651.

Rozanski, A., Blumenthal, J. A., and Kaplan, J. (1999). Impact of psychological factors on the pathogenesis of cardiovascular disease and implications for therapy. Circulation 99, 2192-2217.

Steptoe, A., Dockray, S., and Wardle, J. (2009). Positive affect and psychobiological processes relevant to health. J. Pers. 77, 1747-1776. 
Clark et al.

Latent constructs in psychosocial factors

Steptoe, A., O’Donnell, K., Marmot, M., and Waddle, J. (2008). Positive affect and psychosocial processes related to health. Br. J. Psychol. 99, 211-227.

Suarez, E. C. (2003). Plasma interleukin-6 is associated with psychological coronary risk factors: moderation by use of multivitamin supplements. Brain Behave. Immune. 17, 296-303.

Suarez, E. C. (2006). Sex differences in the relation of depressive sumptoms, hostility, and anger expression to indices of glucose metabolism in nondiabetic adults. Health Psychol. 25, 484-492.

Suls, J., and Bunde, J. (2005). Anger, andiety, and depression as risk factors for cardiovascular disease: the problems and implications of overlapping affective dispositions. Psychol. Bull. 131, 260-300.

Taylor, S. E., Lehman, B. J., Kiefe, C. I., and Seeman, T. E. (2006). Belationship of early life stress and psychological functioning to adult C-reactive protein in the coronary artery risk development in young adults study. Biol. Psychiatry 60, 819-824.

Watson, D., and Clark, L. A. (1984). Negative affectivity: the disposition to experience aversive emotional states. Psychol. Bull. 96, 465-490.

Whitfield, K. E., Weidner, G., Clark, R., and Anderson, N. B. (2002). Sociodemographic diversity and behavioral medicine. J. Consult. Chin. Psychol. 70, 463-481.

Williams, D., Yo, Y., Jackson, J., and Anderson, N. (1997a). Racial differences in physical and mental health. Socio-economic status, stress and discrimination. J. Health Psychol. 2, 335-351.

Williams, R. B., Barefoot, J. C., Blumenthat, J. A., Helms, M. J., Luecken, L., Peeper, C. F., Siegler, I. C., and Suarez, E. C. (1997b). Psychosocial correlates of job strain in a sample of working women. Arch. Gen. Psychiatry 54, 543-548.

Wilson, R. S., Bienias, J. L., Mendes De Leon, C. F., Evans, D. A., and Bennett,
D. A. (2003). Negative affect and mortality in older persons. Am. J. Epidemiol. 158, 827-835.

Wilson, R. S., Kruger, K. R., Gu, L., Bienias, J. L., Menders De Leon, C. F., and Evans, D. A. (2005). Neuroticism, extraversion, and nortality in a defined population of older persons. Psychosom. Med. 67, 841-845.

Wu, A. D., Li, Z., and Zumbo, B. D. (2007). Decoding the meaning of factorial invariance and updating the practice of multi-group confirmatory factor analysis: a demonstration with TIMSS data. Pratt. Assess. Res. Evil. 12, 1-26.

Yuan, K.-H., and Bentler, P. M. (2000). Three likelihood-based methods for mean and covariance strucktore analysis with nonnormal missing data. Sociol. Methodol. 30, 165-2000.

Conflict of Interest Statement: The authors declare that the research was conducted in the absence of any commercial or financial relationships that could be construed as a potential conflict of interest.

Received: 12 August 2011; accepted: 17 January 2012; published online: 02 February 2012.

Citation: Clark CJ, Henderson KM, de Leon CFM, Gus H, Lunos S, Evans DA and Everson-Rose SA (2012) Latent constructs in psychosocial factors associated with cardiovascular disease: an examineion by race and sex. Front. Psychiatry 3:5. do: 10.3389/fpsyt.2012.00005 This article was submitted to Frontiers in Affective Disorders and Psychosomatic Research, a specialty of Frontiers in Prychiatry.

Copyright (c) 2012 Clark, Henderson, de Leon, Gus, Lunos, Evans and EversonRose. This is an open-access article distribute under the terms of the Creative Commons Attribution Non Commercial License, which permits non-commercial use, distribution, and reproduction in other forums, provided the original authors and source are credited.

www.frontiersin.org

February 2012 | Volume 3 | Article 5 | 9 